\title{
Prevalencia de desórdenes gastrointestinales funcionales y hábito intestinal en lactantes menores de 12 meses internados en el Hospital Infantil Baca Ortiz de Quito, Ecuador
}

\author{
Edgar Játiva-Mariño* \\ Zahira Játiva-Cabezas** \\ Carlos Alberto Velasco-Benítez ${ }^{* * *}$
}

\begin{abstract}
*Médico. Pediatra. Profesor. Universidad Central del Ecuador. Quito, Ecuador. Universidad de La Frontera, Temuco, Chille.
**Médico y Cirujano. Hospital Infantil Baca Ortíz. Quito, Ecuador.

***Médico. Gastroenterólogo pediatra. Profesor. Universidad del Valle. Cali, Colombia

Correspondencia: Dr. Carlos Velasco. Dirección: Calle 5 D 38 A 35 Torre 1 Consultorio 626 Cali, Colombia. Teléfono:+57 25545226. Correo electrónico: carlos.velasco@correounivalle.edu.co
\end{abstract}

\section{Resumen}

Introducción: la prevalencia de desórdenes gastrointestinales funcionales en niños menores ecuatorianos se desconoce y no hay una prueba estándar para evaluar la consistencia de las heces. Objetivo: determinar la prevalencia de desórdenes gastrointestinales funcionales en lactantes menores a 12 meses e identificar el hábito intestinal. Metodología: estudio de prevalencia en lactantes internados del Hospital Infantil Baca Ortíz de Quito, Ecuador, a quienes se les diagnosticó diarrea funcional, cólico, estreñimiento funcional, disquecia, regurgitación, síndrome de vómito cíclico y rumiación. Fueron incluidas variables sociodemográficas, clínicas y preguntas sobre hábitos intestinales, basados en el Cuestionario para Síntomas Digestivos Pediátricos Roma III traducido y validado en español. Se hizo análisis uni y multivariados y el cálculo de los ORs, siendo una $p<0,05$ significativa. Resultados: fueron incluidos 147 lactantes, de 6,8 $\pm 3,3$ meses de edad, 50,3\% niñas. La principal causa de hospitalización fue del sistema respiratorio. Se reportó 36,0\% con algún desorden gastrointestinal funcional: cólico (12,2\%), estreñimiento funcional (9,5\%), disquecia (8,0\%) y regurgitación (7,5\%). No hubo factores de riesgo asociados. Se reportaron heces duras en 4,1\% y 7,4\% por Criterios de Roma III y Escala de Bristol, respectivamente. Hubo concordancia aceptable (kappa=0,3989, $p=0,0221$ ) entre el reporte de los padres por los Criterios de Roma III y la Escala de Bristol. Conclusión: un tercio de estos niños presentó algún desorden gastrointestinal funcional, siendo los más frecuentes: estreñimiento funcional, regurgitación, cólico y disquecia; sin posibles factores de riesgo; identificándose una concordancia aceptable entre lo reportado por los padres según los Criterios de Roma III y la Escala de Bristol. MÉD.UIS.2019;32(2):13-21

Palabras claves: Enfermedades del sistema digestivo. Lactante. Estreñimiento. Cólico. Reflujo gastroesofágico.

\section{Prevalence of functional gastrointestinal disorders and intestinal habit hospitalized in infants under 12 months of hospital infantil Baca Ortiz from Quito, Ecuador}

Abstract

Introduction: the prevalence of functional gastrointestinal disorders in Ecuadorian younger infant is unknown and there is no gold standard for stool consistency. Objective: to determine the prevalence of functional gastrointestinal disorders in infants younger 12 months and to identify the intestinal habit. Methodology: prevalence study in hospitalized infants at the Hospital Infantil Baca Ortíz in Quito, Ecuador, who were diagnosed with functional diarrhea, colic, functional constipation, dyschezia, regurgitation, cyclic vomiting syndrome and rumination. Socio-demographic, clinical variables and questions about intestinal habits, based on the Questionnaire for Pediatric Digestive Symptoms Roma III translated and validated in Spanish, were included. The statistic included uni and multivariate analyzes and the calculation of the ORs, being a significant $p<0.05$. Results: a total of 147 infants, $6,8 \pm 3,3$ months old, 50,3\% girls; with the

Artículo recibido el 14 de mayo del 2018 y aceptado para publicación el 2 de junio del 2019. 
main cause of hospitalization the respiratory system. Were reported 36.0\% with some functional gastrointestinal disorders: colic (12.2\%), functional constipation (9,5\%), dyschezia $(8,0 \%)$ and regurgitation $(7,5 \%)$. There were no possible associated risk factors. Hard stools were reported in 4,1\% and 7,4\% by Criteria of Rome III and Bristol Stool Scale, respectively. There was acceptable agreement (kappa =0,3989, p = 0.0221 ) between the parents' report for the Rome Criteria III and the Bristol Stool Scale. Conclusion: one third of these children presented some functional gastrointestinal disorders, being the most frequent functional constipation, regurgitation, colic and dyschezia; without possible risk factors; identifying an acceptable concordance between what was reported by parents according to Rome Criteria III and Bristol Stool Scale. MÉD.UIS.2019;32(2):13-21

Keywords: Digestive system diseases. Infant. Constipation. Colic. Gastroesophageal reflux.

¿Cómo citar este artículo?: Játiva-Mariño E, Játiva-Cabezas Z, Velasco-Benítez CA. Prevalencia de desórdenes gastrointestinales funcionales y hábito intestinal en lactantes menores de 12 meses internados en el Hospital Infantil Baca Ortíz De Quito, Ecuador. MÉD. UIS.2019;32(2):13-21. doi $10.18273 /$ revmed.v32n2-2019002

\section{Introducción}

Los síntomas gastrointestinales son una causa común de consulta en niños menores de 4 años y solo en el $10 \%$ de los casos se le encuentra una causa orgánica al síntoma'. Según los Criterios de Roma IV, los desórdenes gastrointestinales funcionales (DGFs) en lactantes y pre-escolares, son una combinación diversa y variable de síntomas gastrointestinales recurrentes o crónicos que luego de una adecuada evaluación médica, no son atribuibles a otras condiciones médicas ${ }^{2}$. Los principales DGFs en lactantes y pre-escolares son el cólico del lactante, la disquecia del lactante, la regurgitación del lactante, el síndrome de rumiación del lactante, el síndrome de vómito cíclico, la diarrea funcional y el estreñimiento funcional ${ }^{3}$. En una muestra representativa de la comunidad, los DGFs se presentan en cerca a un tercio de lactantes y pre-escolares norteamericanos ${ }^{4}$; en Europa y Asia ha sido reportada una prevalencia para DGFs en lactantes y pre-escolares hasta del 38,0\%, y en niños entre 1 y 4 años de edad de Ecuador, Panamá y Nicaragua, la prevalencia de presentar algún DGFs es aproximadamente del $12,0 \%{ }^{5}$ y en lactantes y pre-escolares colombianos, esta prevalencia es del 40,5\% ${ }^{6}$. En Estados Unidos de 1997 a 2009, estos DGFs ha implicado mayores hospitalizaciones (de 6'348 537 a 6’393 803) y costos (de US\$6115 a US\$18 058). Un mayor conocimiento sobre la epidemiología de estos DGFs, evitarán hospitalizaciones innecesarias y pruebas de diagnóstico potencialmente dañinas. La identificación de los DGFs es el primer paso para mejorar su manejo en la edad pediátrica ${ }^{8}$.

Se desconoce la prevalencia de DGFs en lactantes y pre-escolares ecuatorianos; la prevalencia de DGFs en escolares y adolescentes ambulatorios de Quito, Ecuador ha sido reportada en un 22,8\%, siendo el estreñimiento funcional (EF) en un $11,8 \%$ y el síndrome de intestino irritable (SII) en un $4,8 \%$, los más frecuentes"; al igual que en otros países latinoamericanos ${ }^{10-14}$. Así mismo, son pocos los estudios que buscan las posibles causas de los DGFs; algunos de ellos identifican la baja calidad de $v^{v i d a}{ }^{4}$, el incremento de consultas médicas por esta causa ${ }^{4}$ y eventos a edad temprana de la vida ${ }^{15}$. De allí la importancia de identificar otras variables que involucren genética, nutrición, ambiente, cultura, infecciones y procesos psicosociales y económicos, entre otros.

La consistencia de las heces es una preocupación frecuente de los cuidadores de niños quienes frecuentemente lo asocian a enfermedad. Actualmente no hay un estándar de oro para evaluar la consistencia de las heces en lactantes y preescolares; Koppen et $\mathrm{al}^{16}$, reportan una concordancia aceptable $(k=0,335 ; p<0,001)$ entre la Escala de Bristol (EB) y el reporte de los padres según los Criterios de Roma III.

El principal objetivo de este estudio es describir la prevalencia y las posibles asociaciones de DGFs en lactantes ecuatorianos menores de 12 meses del Hospital Infantil Baca Ortíz de Quito, por medio de los Criterios de Roma III en español; y como objetivo específico, identificar el hábito intestinal y la concordancia entre la EB y el reporte de los padres para DGFs.

\section{Materiales y métodos}

Se realizó un estudio descriptivo observacional no experimental tipo prevalencia en lactantes ecuatorianos menores de 12 meses de edad quienes se encontraban internados por diversas causas en el 
Hospital Infantil Baca Ortíz de Quito, Ecuador entre el 1 de julio y 31 de octubre de 2015.

El tipo de muestreo fue intencional o de conveniencia. Se incluyeron niños de cualquier género entre los 1 y 12 meses de edad, en quienes sus padres o tutores aceptaron su participación en el estudio mediante la forma de un consentimiento escrito; y fueron excluidos, niños con enfermedades digestivas orgánicas diagnosticadas previamente, como enfermedad por reflujo gastroesofágico, estreñimiento crónico orgánico, síndrome de intestino corto, atresia esofágica, enfermedad de Hisrschsprung, entre otras.

El Cuestionario para Síntomas Gastrointestinales Pediátricos de los Criterios de Roma III (QPGS-III) es un cuestionario traducido y validado al español compuesto por 29 ítems, designado para diagnosticar desórdenes gastrointestinales funcionales (DGFs) en niños. El QPGS-III fue realizado sobre eventos presentes en el momento de la encuesta. Las preguntas revisan la localización, frecuencia y severidad de los síntomas gastrointestinales, así como la discapacidad relacionada y los síntomas somáticos. Para este estudio, el QPGS-III para lactantes y pre-escolares, fue traducido del inglés al español por 2 gastroenterólogos pediatras de Colombia con dominio de ambos idiomas ${ }^{17}$. Para asegurar la comprensión, la redacción del cuestionario se adaptóa la lengua local entrevistando a algunos padres o tutores de niños hospitalizados, estando todos de acuerdo en la versión final y manifestando comprensión de las preguntas del QPGS-III para lactantes y pre-escolares en español. El diagnóstico de DGFs se realizó luego de aplicar la encuesta para Síntomas Gastrointestinales Pediátricos para Lactantes y Pre-escolares de los Criterios de Roma III en español, siendo dichos DGFs la diarrea funcional (DF), el cólico (en $\leq 4$ meses), el estreñimiento funcional (EF), la disquecia (en $\leq$ 5 meses), la regurgitación, el síndrome de vómito cíclico (SVC) y la rumiación. Los padres o tutores de los niños internados fueron invitados a participar en el estudio y quienes aceptaban firmando un consentimiento informado. EI QPGS-III fue realizado a los Cuidadores de los niños de 0 a 12 meses, entre el 1 de julio y el 31 de octubre de 2015 por uno de los coinvestigadores (ZJC), quien fue previamente entrenada y capacitada para la realización del QPGSIII y quien además fue la encargada de contactar a cada familiar particpante. Se diseñó una base de datos en el programa Excel en la que se ingresó la información recolectada, realizando doble digitación para el ingreso de los datos. Los datos posteriormente fueron exportados a Stata 15 para su análisis.

Fueron incluidas variables sociodemográficas (sexo, edad), familiares (padres separados/divorciados, hijo único), y clínicas (diarrea previa, hábitos intestinales). Las preguntas sobre hábitos intestinales incluyeron 1) tipo de las heces según la Escala de Bristol (EB) (tipos 1 al 7), 2) frecuencia, consistencia y tamaño de las heces, y 3) síntomas asociados como dolor al defecar y maniobras de retención de heces. Las características de las heces son las que presentaban los niños en el momento de la encuesta.

Para identificar la posibilidad de errores de transcripción, el 10\% de los datos fueron revisados y comparados con las formas originales. El estudio fue aprobado por el Comité de Ética de la Universidad del Valle de Cali, Colombia y autorizado a realizar por la Dirección General del Hospital Infantil Baca Ortíz de Quito, Ecuador.

Análisis estadístico. Para determinar la prevalencia y sus posibles factores de riesgo, los datos fueron analizados usando una t-student a dos colas, chi cuadrado, y prueba exacta de Fisher (Stata 10 software; StataCorp, College Station, Texas). Para los posibles factores de riesgo de DGFs, se hicieron análisis uni y multivariados y el cálculo de los ORs fueron realizados entre la variable exposición de interés (sexo, características familiares) y la variable efecto (presencia o ausencia de DGFs). El valor de una $\mathrm{p}<0,05$ fue considerado como estadísticamente significativo. Para ver el grado de concordancia entre la consistencia de las heces reportado por los padres según los Criterios de Roma III y la EB, se usó el coeficiente de Kappa (k) y sus niveles de concordancia. En el caso del reporte de los padres según los Criterios de Roma III fue excluida la respuesta "variable", debido a que no podía ser asignada a alguno de los grupos de consistencia de heces predefinido.

\section{Resultados}

Fueron invitados 180 padres de lactantes menores de 12 meses que se encontraban internados por diversas causas entre el 1 de julio y el 31 de octubre de 2015 en el Hospital Infantil Baca Ortíz de Quito, Ecuador; de los cuales 170 fueron enrolados $(94,4 \%)$ y posteriormente 23 fueron excluidos por mal diligenciamiento de las 
encuestas (12,8\%), obteniéndose una muestra final de 147 (87,1\%). Ningún niño presentó enfermedades digestivas orgánicas diagnosticadas previamente. La consistencia interna, estabilidad y equivalencia de la Encuesta de los Criterios de Roma III Pediátrica en español para identificar los DGFs en este grupo de lactantes ecuatorianos hospitalizados, presentó un coeficiente de confiabilidad moderado (a de conbrach $=0,5365$ ).

De los 147 lactantes analizados, con edad 6,8 $8 \pm 3,3$ meses, 81 (55,1\%) eran lactantes mayores entre $7 \mathrm{y}$ 12 meses de edad; 50,3\% del género femenino; 53,1\% hijos únicos; 19,0\% con padres separados/divorciados; $20,4 \%$ con antecedente de diarrea previa en el último año, y la causa de hospitalización más frecuente por compromiso del sistema respiratorio en el 34,3\% (Ver Tabla 1).

Tabla 1. Características sociodemográficas y clinicas de lactantes menores de 12 meses internados en el Hospital Infantil Baca Ortíz de Quito, Ecuador ( $N=147)$

\begin{tabular}{|c|c|c|}
\hline & $n$ & $\%$ \\
\hline \multicolumn{3}{|l|}{ Grupo de edad } \\
\hline $\begin{array}{l}\text { Lactante menor (0-6 } \\
\text { meses) }\end{array}$ & 66 & 44,9 \\
\hline $\begin{array}{l}\text { Lactante mayor (7- } \\
12 \text { meses) }\end{array}$ & 81 & 55,1 \\
\hline \multicolumn{3}{|l|}{ Sexo } \\
\hline Femenino & 74 & 50,3 \\
\hline Masculino & 73 & 49,7 \\
\hline \multicolumn{3}{|c|}{ Antecedentes familiares } \\
\hline \multicolumn{3}{|l|}{ Hijo único } \\
\hline No & 69 & 46,9 \\
\hline $\mathrm{Si}$ & 78 & 53,1 \\
\hline \multicolumn{3}{|c|}{ Padres separados/divorciados } \\
\hline No & 119 & 81,0 \\
\hline $\mathrm{Si}$ & 28 & 19,0 \\
\hline \multicolumn{3}{|c|}{ Antecedente diarrea previa } \\
\hline No & 117 & 79,6 \\
\hline $\mathrm{Si}$ & 30 & 20,4 \\
\hline \multicolumn{3}{|c|}{ Diagnóstico de hospitalización } \\
\hline Respiratorio & 50 & 34,3 \\
\hline Renal & 30 & 20,0 \\
\hline Hematológico & 17 & 11,4 \\
\hline Quirúrgico & 13 & 8,6 \\
\hline Ortopédico & 13 & 8,6 \\
\hline Cardíaco & 8 & 5,7 \\
\hline Dermatológico & 8 & 5,7 \\
\hline Nervioso & 8 & 5,7 \\
\hline
\end{tabular}

Fuente: autores
Cincuenta y tres lactantes menores de 12 meses presentaron algún DGFs (36,0\%), siendo el cólico (por definición entre los 1 y 4 meses de edad) el más frecuente (12,2\%), seguido del estreñimiento funcional (EF) (9,5\%), la disquecia (por definicion entre los 1 y 5 meses de edad) (8,0\%), la regurgitación $(7,5 \%)$, la rumiación $(6,1 \%)$, el SVC $(5,4 \%)$ y la diarrea funcional $(1,4 \%)$. De los 53 lactantes con algún DGFs, en once $(20,8 \%)$ se presentaron más de dos DGFs: en $6(11,3 \%)$ se presentaron dos DGFs (tres con regurgitación, rumiación, y uno con EF, regurgitación, con EF, SVC y SVC, rumiación, respectivamente) y en cinco (9,5\%) se presentaron tres DGFs (dos con cólico, disquecia, rumiación y disquecia, regurgitación, rumiación, respectivamente y una con regurgitación, SVC, rumiación (Ver Tabla 2).

Tabla 2. Prevalencia de desórdenes gastrointestinales funcionales de lactantes menores de 12 meses internados en el Hospital Infantil Baca Ortíz de Quito, Ecuador ( $n=147)$

\begin{tabular}{|l|c|}
\hline & $\mathbf{n}(\%)$ \\
\hline Sin desórdenes gastrointestinales funcionales & $94(64,0)$ \\
\hline Con desórdenes gastrointestinales funcionales & $53(36,0)$ \\
\hline \multicolumn{2}{|c|}{ Diagnóstico } \\
\hline $\begin{array}{l}\text { Cólico (por definición en menores de 4 meses) } \\
\text { (n=41) }\end{array}$ & $5(12,2)$ \\
\hline Estreñimiento funcional & $14(9,5)$ \\
\hline $\begin{array}{l}\text { Disquecia (por definición en menores de 5 } \\
\text { meses) (n=50) }\end{array}$ & $4(8,0)$ \\
\hline Regurgitación & $11(7,5)$ \\
\hline Rumiación & $9(6,1)$ \\
\hline Síndrome de vómito cíclico & $8(5,4)$ \\
\hline Diarrea funcional & $2(1,4)$ \\
\hline $\begin{array}{l}\text { Superposición de 2 o más desórdenes } \\
\text { gastrointestinales funcionales (n=53) }\end{array}$ & $11(20,8)$ \\
\hline Regurgitación + Rumiación & $1(1,9)$ \\
\hline Estreñimiento funcional + Regurgitación & $2(3,8)$ \\
\hline $\begin{array}{l}\text { Estreñimiento funcional + Síndrome de vómito } \\
\text { cílico }\end{array}$ & $1(1,9)$ \\
\hline Síndrome de vómito cículico + Rumiación \\
\hline Cólico + Disquecia + Rumiación \\
\hline Disquecia + Regurgitación + Rumiación \\
\hline Regurgitación + Síndrome de vómito cíclico + \\
\hline Rumiación
\end{tabular}

Fuente: autores 
Mayo - Agosto

Hubo mayor oportunidad de presentar DGFs en los lactantes menores de 12 meses quienes eran hijos únicos, con padres separados/divorciados y con el antecedente de diarrea 1 año previo, pero sin diferencias significativas ( $p>0,05)$ (Ver Tabla 3 ).

Con relación a la consistencia de las heces, 6 padres (4,1\%) de los lactantes menores de 12 meses, reportaron según los Criterios de Roma III, heces duras o muy duras y siete padres (7,4\%) según la EB (tipo 1 y 2 ), heces duras. $65(44,2 \%)$ y 46 padres $(48,5 \%)$ describieron las heces como normales según los Criterios de Roma
III y EB (tipos 3, 4 y 5), respectivamente. Según los Criterios de Roma III, 71 padres (48,3\%) reportaron las heces blandas, muy blandas, mucosas o líquidas y según EB (tipos 6 y 7), 42 padres (52,3\%), líquidas. El $18,4 \%, 10,2 \%$ y $2,7 \%$ de los padres según los Criterios de Roma III, describieron en los lactantes menores de 12 meses, dolor al defecar, heces grandes y retención de heces, respectivamente. En cuanto a la frecuencia de las deposiciones, solo cinco $(3,4 \%)$ de los padres reportaron según los Criterios de Roma III, dos o menos deposiciones por semana en los lactantes menores de 12 meses (Ver Tabla 4).

Tabla 3. Asociación entre desórdenes gastrointestinales funcionales y variables sociodemográficas y familiares en lactantes menores de 12 meses internados en el Hospital Infantil Baca Ortíz de Quito, Ecuador ( $n=147$ )

\begin{tabular}{|c|c|c|c|c|c|}
\hline & \multicolumn{2}{|c|}{$\begin{array}{c}\text { Desórdenes gastrointestinales } \\
\text { funcionales }\end{array}$} & \multirow{2}{*}{ OR } & \multirow{2}{*}{ IC95\% } & \multirow{2}{*}{$\mathbf{p}$} \\
\hline & $\begin{array}{l}\text { Presentes } \\
53(36,0 \%)\end{array}$ & $\begin{array}{l}\text { Ausentes } \\
94(64,0 \%)\end{array}$ & & & \\
\hline \multicolumn{6}{|c|}{ Variables sociodemográficas } \\
\hline \multicolumn{6}{|l|}{ Grupo de edad } \\
\hline Lactante menor (0-6 meses) & 25 & 41 & 1,00 & & \\
\hline Lactante mayor (7-12 meses) & 28 & 53 & 0,86 & $0,41-1.80$ & 0,67 \\
\hline \multicolumn{6}{|l|}{ Sexo } \\
\hline Femenino & 31 & 43 & 1,00 & & \\
\hline Masculino & 22 & 51 & 0,59 & $0,28-1,24$ & 0,13 \\
\hline \multicolumn{6}{|c|}{ Variables familiares } \\
\hline \multicolumn{6}{|l|}{ Hijo único } \\
\hline No & 24 & 45 & 1,00 & & \\
\hline Si & 29 & 49 & 1,10 & $0,53-2,30$ & 0,76 \\
\hline \multicolumn{6}{|c|}{ Padres separados/divorciados } \\
\hline No & 41 & 78 & 1,00 & & \\
\hline $\mathrm{Si}$ & 12 & 16 & 1,42 & $0,55-3,56$ & 0,40 \\
\hline \multicolumn{6}{|c|}{ Antecedente diarrea previa } \\
\hline No & 42 & 75 & 1.00 & & \\
\hline Si & 11 & 19 & 1,03 & $0,40-2,54$ & 0,93 \\
\hline
\end{tabular}

Fuente: autores

Para el análisis de la concordancia entre el reporte de los padres por los Criterios de Roma III y la EB, fueron excluidos los cinco padres que respondieron "heces variables" según los Criterios de Roma III. El nivel de concordancia se basó en el Coeficiente de Kappa (k): pobre $(0,00)$, leve $(0,01-0,20)$, aceptable $(0,21-0,40)$, moderada $(0,41-0,60)$, buena $(0,61-0,80)$, excelente $(0,81-0,99)$ y perfecta $(1,00)$. En general, la concordancia fue aceptable $(k=0,3989, p=0,0221)$ entre el reporte de los padres por los Criterios de Roma III y la EB: hubo una concordancia perfecta cuando se analizaron los lactantes menores de 12 meses con EF ( $k=1,000$, $\mathrm{p}=0,0041)$; buena al analizar la consistencia de las heces duras $(k=0,7179, p=0,0000)$, y moderada $(k=0,4355$, $p=0,0005)$ y aceptable $(k=0,3467, p=0,0016)$, al revisar el grupo de edad de los lactantes menores entre $1 \mathrm{y}$ 6 meses y los lactantes mayores entre 7 y 12 meses, respectivamente (Ver Tabla 5). 
Játiva-Mariño E, Játiva-Cabezas Z, Velasco-Benítez CA.

Tabla 4. Hábito intestinal en lactantes menores de 12 meses internados en el Hospital Infantil Baca Ortíz de Quito, Ecuador $(n=147)$

\begin{tabular}{|c|c|c|c|c|}
\hline & $\begin{array}{c}\text { Total } \\
1-12 \\
\text { meses } \\
(\mathrm{N}=147) \\
\mathrm{n}(\%)\end{array}$ & $\begin{array}{c}\text { Lactante } \\
\text { menor } \\
1-6 \\
\text { meses } \\
\begin{array}{c}(n=66) \\
n(\%)\end{array}\end{array}$ & 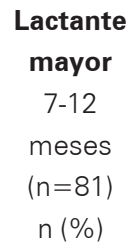 & $\mathbf{p}$ \\
\hline \multicolumn{5}{|c|}{ Frecuencia deposición } \\
\hline $\begin{array}{l}<2 x \\
\text { semana }\end{array}$ & $5(3,4)$ & $2(3,0)$ & $3(3,7)$ & 0,596 \\
\hline $\begin{array}{l}3-6 x \\
\text { semana }\end{array}$ & $16(10,9)$ & $8(12,1)$ & $8(9,9)$ & 0,431 \\
\hline Diario & $46(31,3)$ & $23(34,9)$ & $23(28,4)$ & 0,254 \\
\hline $2-3 \times$ dia & $71(48,3)$ & $27(40,9)$ & $44(54,3)$ & 0,073 \\
\hline$>3 \times$ dia & $9(6,1)$ & $6(9,1)$ & $3(3,7)$ & 0,157 \\
\hline \multicolumn{5}{|c|}{ Consistencia según Roma III } \\
\hline $\begin{array}{l}\text { Dura o muy } \\
\text { duras }\end{array}$ & $6(4,1)$ & $0(0,0)$ & $6(7,4)$ & 0,026 \\
\hline Normales & $65(44,2)$ & $19(28,8)$ & $46(56,8)$ & 0,001 \\
\hline $\begin{array}{l}\text { Blandas o } \\
\text { muy blandas }\end{array}$ & $47(32,0)$ & $28(42,4)$ & $19(23,5)$ & 0,011 \\
\hline Mucosa & $4(2,7)$ & $3(4,6)$ & $1(1,2)$ & 0,237 \\
\hline Líquidas & $20(13,6)$ & $14(21,2)$ & $6(7,4)$ & 0,014 \\
\hline Variable & $5(3,4)$ & $2(3,0)$ & $3(3,7)$ & 0,596 \\
\hline \multicolumn{5}{|c|}{ Consistencia según Bristol } \\
\hline Tipo 1 & $3(3,2)$ & $2(4,6)$ & $1(2,0)$ & 0,423 \\
\hline Tipo 2 & $4(4,2)$ & $2(4,6)$ & $2(3,9)$ & 0,609 \\
\hline Tipo 3 & $9(9,5)$ & $3(6,8)$ & $6(11,8)$ & 0,359 \\
\hline Tipo 4 & $24(25,3)$ & $8(18,2)$ & $16(31,4)$ & 0,154 \\
\hline Tipo 5 & $13(13,7)$ & $6(13,6)$ & $7(13,7)$ & 0,574 \\
\hline Tipo 6 & $27(28,4)$ & $13(29,6)$ & $14(27,5)$ & 0,434 \\
\hline Tipo 7 & $15(15,8)$ & $10(22,7)$ & $5(9,8)$ & 0,065 \\
\hline \multicolumn{5}{|c|}{ Dolor al defecar } \\
\hline No & $120(81,6)$ & $57(86,4)$ & $63(77,8)$ & 0,13 \\
\hline $\mathrm{Si}$ & $27(18,4)$ & $9(13,6)$ & $18(22,2)$ & \\
\hline \multicolumn{5}{|c|}{ Heces grandes } \\
\hline No & $132(89,8)$ & $62(93,9)$ & $70(86,4)$ & 0,109 \\
\hline Si & $15(10,2)$ & $4(6,1)$ & $11(13,6)$ & \\
\hline \multicolumn{5}{|c|}{ Retención de heces } \\
\hline Nunca & $127(86,4)$ & $61(92,4)$ & $66(81,5)$ & 0,068 \\
\hline $\begin{array}{l}\text { De vez en } \\
\text { cuando }\end{array}$ & $16(10,9)$ & $4(6,1)$ & $12(14,8)$ & \\
\hline
\end{tabular}

Fuente: autores
MÉD.UIS. 2019;32(2):13-21

Tabla 5. Fuerza de concordancia en lactantes menores de 12 meses internados en el Hospital Infantil Baca Ortíz de Quito, Ecuador $(n=147)$

\begin{tabular}{|l|l|l|l|}
\hline \multicolumn{1}{|c|}{ Variable } & \multicolumn{1}{c|}{ kappa } & p & Interpretación \\
\hline Según cuestionario & 0,3989 & 0,0221 & Aceptable \\
\hline $\begin{array}{l}\text { Roma III vs } \\
\text { Bristol }\end{array}$ & 0,4355 & 0,0005 & Moderada \\
\hline Según grupos de edad \\
\hline $1-6$ meses & 0,456 & 0,0016 & Aceptable \\
\hline 7-12 meses & 0,3467 & \\
\hline Según desorden gastrointestinal funcional \\
\hline $\begin{array}{l}\text { 1-12 meses con } \\
\text { estreñimiento }\end{array}$ & 1,0000 & 0,0041 & Perfecta \\
\hline Según consistencia de las heces \\
\hline Duras & 0,7179 & 0,0000 & Buena \\
\hline
\end{tabular}

Fuente: autores

\section{Discusión}

Los profesionales de la salud dependen de los síntomas reportados e interpretados por los padres de lactantes y pre-escolares para diferenciar entre salud y enfermedad, que involucra tanto al niño como a sus familiares. En el caso de los lactantes y preescolares, los Criterios de Roma III ${ }^{18}$ y IV ${ }^{2}$, por medio del QPGS, que consideran una combinación variable de síntomas crónicos o recurrentes, a menudo dependientes de la edad, que no son explicados por anormalidades estructurales o bioquímicas, no orgánicos, se logra determinar los DGFs, que incluyen, entre otros, regurgitación, síndrome de rumiación, síndrome de vómito cíclico, cólico, diarrea funcional, disquecia y EF.

La consistencia interna, estabilidad y equivalencia del QPGS-III para lactantes y pre-escolares en este grupo de lactantes ecuatorianos hospitalizados menores de 12 meses, demostró un coeficiente de confiabilidad moderado, que se puede considerar como aceptable al compararlo con el alfa de conbrach de 0,71 en lactantes y pre-escolares colombianos para el mismo fin ${ }^{17}$.

En este estudio de lactantes ecuatorianos hospitalizados menores de 12 meses de edad, la prevalencia para identificar al menos un DGFs por medio del QPGS-III para lactantes y pre-escolares fue del 36,0\%; siendo relativamente similar a la reportada en lactantes colombianos ambulatorios menores de 12 meses de edad $(46,1 \%)^{6}$. En niños 
norteamericanos, van Tilburg et al $^{19}$. Al desarrollar y validar el cuestionario para identificar DGFs en padres de 332 lactantes y pre-escolares ambulatorios, encontraron por el reporte de los padres en $87(26,2 \%)$ lactantes menores de 12 meses algún DGF; Rouster et $\mathrm{al}^{20}$. En 332 lactantes y pre-escolares ambulatorios describen por medio de un nuevo cuestionario, 172 niños $(51,8 \%)$ con algún DGFs, y van Tilburg et $a^{4}$. Al entrevistar 300 madres de niños ambulatorios entre o y 3 años de edad por medio de los Criterios de Roma III, identificaron un $27.0 \%$ de algún DGFs, estando asociados a una más baja calidad de vida y a incremento de consultas médicas en los lactantes y pre-escolares que presentaban algún DGFs. Ferreira-Maia et al. ${ }^{21}$, en una revisión sistemática sobre DGFs en lactantes y pre-escolares, donde se incluyen 12 estudios escritos en inglés y uno en chino; publicados entre 2004 y 2015; ocho europeos, tres americanos y dos asiáticos; nueve de prevalencia y cuatro prospectivos; relatan una prevalencia entre el $27,1 \%$ y $38 \%$ para presentar algún DGFs por Criterios de Roma. La posible explicación en la variación de estas prevalencias involucra conceptos genéticos, nutricionales, ambientales, psicosociales, culturales, socioeconómicos e infecciosos, entre otros, muy propios de cada región; además que los lactantes ecuatorianos fueron niños hospitalizados a diferencia de los restantes estudios que reportan niños ambulatorios.

Si no se toman en cuenta el cólico y la disquecia, que se presentan en menores de 4 y 5 meses de edad, respectivamente; las principales causas de DGFs en lactantes hospitalizados ecuatorianos menores de 12 meses fueron el EF (9,5\%) y la regurgitación (7,5\%); entidades igualmente frecuentes en los lactantes colombianos ambulatorios menores de 12 meses de edad con un 16,1\% para EF y un 8,0\% para regurgitación ${ }^{6}$. Rouster et $\mathrm{al}^{20}$, en 332 lactantes y pre-escolares ambulatorios de 1,2 $\pm 0,9$ años, $52 \%$ del género masculino, $57 \%$ blancos y $33 \%$ afroamericanos; describen como las principales causas de DGFs, el EF en el $29,2 \%$ y la regurgitación en el $13,3 \%$. FerreiraMaia et $a^{21}$, en su revisión sistemática sobre DGFs en lactantes y pre-escolares, relatan una prevalencia para EF y regurgitación entre un $0,68 \%-31,0 \%$ y un $0,44 \%$ $25,9 \%$, respectivamente. van Tilburg et al. 4,19 en dos reportes identifican primero a la regurgitación (25,9\%$51,9 \%)$ y luego al EF (4,7\%-36,7\%) como principales causas de DGFs en lactantes y pre-escolares ambulatorios. Con relación a la regurgitación, el 24\% de los niños entre los 4 y 6 meses de edad, regurgitan $\geq$ cuatro veces al día; debido altos volúmenes de alimentos líquidos, a la inmadurez de la motilidad digestiva y a la posición del lactante; su manejo incluye educación y pocos alimentos y espesados, y en el 90\% de los casos resuelve alrededor de los 12 meses de edad 3 . La rumiación, que se presentó en el $6,1 \%$ de los lactantes ecuatorianos hospitalizados menores de 12 meses de edad en este estudio, puede ser secundaria a una deprivación sensorial y emocional; y el tratamiento incluye, intervenciones medio ambientales y nutricionales, y alimentación transyeyunal ${ }^{22}$. La diarrea funcional, que fue el DGFs menos frecuente en este grupo de lactantes ecuatorianos hospitalizados menores de 12 meses del estudio, con un $1,4 \%$, es una entidad, secundaria a anormalidades de motilidad y dietarias y cuestionada un incremento en la secreción mucosa; cuyo manejo incluye educación y cambios dietarios, y usualmente resuelve a los 4 años de edad 3 . Con relación al cólico, principal causa de DGFs en menores de 4 meses en este grupo de lactantes ecuatorianos hospitalizados menores de 12 meses, Stetutel et $\mathrm{al}^{23}$, proponen instrumentos validados, resultados y definiciones uniformes para hacer comparación entre los estudios de intervención para cólico infantil, y Szajweska et $\mathrm{al}^{24}$, debido a la falta de una terapia eficaz para el manejo del cólico infantil y la buena seguridad del Lactobacillus reuteri DSM 17938, propone esta opción terapéutica, a ser considerada con los cuidadores del lactante. La disquecia que se presentó en 4/50 (8,0\%) de los lactantes ecuatorianos hospitalizados menores de 5 meses estudiados, es una entidad frecuente, que se piensa sea secundaria a una incoordinación en la dinámica de la defecación; siendo su manejo al educación y tranquilidad a los cuidadores y evitar los estímulos anales; que evoluciona en la mayoría de los casos, a la mejoría alrededor de los 6 y 7 meses de edad ${ }^{22}$. Chogle et al..$^{25}$ en 351 lactantes latinoamericanos, 56,0\% masculinos, de Colombia, Panamá y Nicaragua menores de 12 meses reportan una prevalencia para disquecia del $15,0 \%$.

En 11/53 (20,8\%) lactantes ecuatorianos hospitalizados menores de 12 meses hubo superposición de DGFs; siendo la regurgitación y la rumiación el par más frecuente y el colico, disquecia, rumiación y la disquecia, regurgitación, rumiación; las ternas más frecuentes. Estos datos ecuatorianos son similares en Colombia, donde se reporta en $43 / 213$ (20,2\%) lactantes ambulatorios menores de 12 meses superposición de DGFs; siendo el par más frecuente la rumiación y el EF; y la terna más frecuente la regurgitación, rumación, $\mathrm{EF}^{6}$. Rouster et al. ${ }^{20}$ reportan 31 niños $(9,3 \%)$ con más de 1 DGFs [veintitrés $(6,9 \%)$ 
con 2 DGFs y $8(2,4 \%)$ con 3 DGFs]; siendo los pares más frecuentes el EF y el Sindrome de vómito cíclico y el EF y la regurgitación; y las ternas más frecuentes la regurgitación, EF, disquecia y el cólico, regurgitación, disquecia.

En este estudio de lactantes ecuatorianos hospitalizados menores de 12 meses, no hubo ningún posible factor de riesgo asociado ( $p>0,05)$; sin embargo, Romanello et al. ${ }^{26}$ al investigar una posible asociación entre el antecedente de haber presentado cólico infantil y la presencia de migraña en niños entre los 6 y 18 años de edad, realizan un estudio en 208 niños con migraña (142 sin aura y 66 con sura), 120 con cefalea tensional y 471 controles; y encuentran que los niños con migraña experimentaron más cólico infantil que los niños sin migraña (OR=6,61 4,38-10,0 IC95\% p<0,01). Según Di Lorenzo ${ }^{15}$, varios estudios en animales y humanos han logrado demostrar que existen algunos eventos a edad temprana de la vida hacen más vulnerables a los niños a presentar DGFs, sobre todo las relacionadas con dolor. Von Baeyer et al. ${ }^{27}$ dentro de la fisiopatología del dolor funcional, proponen un daño en los sistemas reguladores del dolor, entre ellos, la prematurez y los eventos traumáticos a tempranas edades. Indrio et al. ${ }^{28}$ en un estudio retrospectivo realizado en niños que consultaron por dolor abdominal recurrente, reportan una historia previa para cólico del $26,4 \%$, de regurgitación del 25,3\% y de EF del 30,1\%, postulando cómo el estrés gastrointestinal en la infancia puede representar una expresión temprana del dolor abdominal relacionado a DGFs.

La concordancia entre el reporte de los padres por los Criterios de Roma III y la EB en este grupo de lactantes ecuatorianos hospitalizados menores de 12 meses, fue aceptable ( $k=0,3989, p=0,0221)$; superando lo reportado en niños colombianos ambulatorios menores de 4 años $(k=0,335, p<001)^{16}$. Van Tilburg et al. ${ }^{19}$ al desarrollar y validar el cuestionario para identificar DGFs en padres de 332 lactantes y pre-escolares ambulatorios, encontraron una concordancia entre leve y sustancial (kappa 0-18-0,76) del cuestionario entre padres y médicos. Bekkali et al. ${ }^{29}$ estudiaron 555 fotografías de heces de 60 recién nacidos a término y 495 pretérminos, dando como resultado la Escala de Amsterdam para determinar la consistencia y apariencia de las heces en recién nacidos a término y pretérmino alimentados con leche materna y con fórmula infantil. En el presente estudio, no se incluyeron preguntas acerca de lactancia materna, fórmulas infantiles y alimentación complementaria, que hubiera permitido identificar la posible asociación con los DGFs y la consistenca de las heces en este grupo de lactantes y pre-escolares ecuatorianos.

La fortaleza de este estudio radica en la metodología sistemática llevada a cabo en investigaciones previas por el Grupo FINDERS (Functional International Digestive Epidemiological Research Survey), que permite comparaciones entre estudios. Entre las limitaciones del estudio está el no haber realizado una encuesta nutricional y el sesgo de selección de los individuos incluidos, pues se trató de niños hospitalizados, datos que no podrán ser extrapolados a la población general, y se debe tener precaución con la discusión aquí expuesta, ya que las comparaciones con estudios previos, son realizadas principalmente a nivel poblacional.

\section{Conclusión}

Una tercera parte de los lactantes ecuatorianos hospitalizados menores de 12 meses, presentaron algún DGFs, siendo los más frecuentes el EF y la regurgitación, sin estar asociados a ningún posible factor de riesgo; identificándose una concordancia aceptable entre lo reportado por los padres según los Criterios de Roma III y la EB.

\section{Referencias bibliográficas}

1. van den Berg M, Benninga M, Di Lorenzo C. Epidemiology of Childhood Constipation: A Systematic Review. Am J Gastroenterol. 2006; 101 (10): 2401-2409

2. Thompson WG. The Road to Rome. Gastroenterology. 2006; 130 (5): 1552-1556

3. Benninga MA, Nurko S, Faure C, Hyman PE, Roberts ISJ, Schechter NL. Childhood Functional Gastrointestinal Disorders: Neonate/Toddler. Gastroenterology. 2016; 150: 1443-1455

4. van Tilburg MAL, Hyman PE, Walker L, Rouster A, Palsson OS, Kim SM, et al. Prevalence of Functional Gastrointestinal Disorders in Infants and Toddlers. J Pediatr. 2015; 166: 684-689

5. Velasco-Benítez CA, Saps M, Chanis R, Játiva E, Mejía M, Moreno $\mathrm{J}$, et al. Desórdenes gastrointestinales funcionales en lactantes y pre-escolares latinoamericanos. Grupo de trabajo de la Sociedad Latinoamericana de Gastroenterología, Hepatología y Nutrición Pediátrica (SLAGHNP). Acta Gastroenterol Latinoam. 2018; 48: 56-64

6. Chogle A, Velasco-Benítez CA, Koppen IJ, Moreno JE, Ramírez CR, Saps M. A Population-Based Study on the Epidemiology of Functional Gastrointestinal Disorders in Young Children. J Pediatr. 2016; 179: 139-143

7. Park R, Mikami S, Leclair J, Bollom A, Lembo C, Sethi S, et al. Inpatient burden of childhood functional GI disorders in the USA: An analysis of national trends in the USA from 1997 to 2009. Neurogastroenterol Motil. 2015; 27 (5): 684-692

8. Benninga M, Candy D, Catto-Smith A, Clayden G, LoeningBaucke V, Di Lorenzo C, et al. The Paris Consensus on Childhood Constipation Terminology (PACCT) Group. JPGN. 2005; 40 (3): 273-275

9. Játiva E, Velasco-Benítez CA, Koppen IJN, Játiva-Cabezas Z, Saps M. Prevalence of Functional Gastrointestinal Disorders in 
Schoolchildren in Ecuador. JPGN. 2016; 63: 25-28

10. Saps M, Nichols-Vinueza DX, Rosen JM, Velasco-Benítez CA. Prevalence of Functional Gastrointestinal Disorders in Colombian School Children. J Pediatr. 2014; 164: 542-545

11. Lu PL, Saps M, Chanis RA, Velasco-Benítez CA. The prevalence of functional gastrointestinal disorders in children in Panama: a school-based study. Acta Pædiatrica. 2016; 105: e232-e236

12. Zablah R, Velasco-Benítez CA, Merlos I, Bonilla S, Saps M. Prevalencia de trastornos funcionales gastrointestinales en niños en edad escolar en El Salvador. Rev Gastroenterol Méx. 2015; 80: 186-191

13. Dhroove G, Saps M, García-Bueno C, Levya Jiménez A, Rodríguez-Reynosa LL, Velasco-Benítez CA. Prevalencia de trastornos gastrointestinales funcionales en escolares mexicanos. Rev Gastroenterol Méx. 2017; 82: 13-18

14. Mejía M, Velasco-Benítez CA, Díaz J. La prevalencia y sus posibles asociaciones de los desórdenes gastrointestinales funcionales en escolares y adolescentes de colegios privados de Managua, Nicaragua. Acta Gastroenterol Latinoam. 2017; 47: 163-168

15. Di Lorenzo C. Impact of Early Life Events on Pediatric Functional Gastrointestinal Disorders. JPGN. 2013; 57 (Suppl 1): S15-S18

16. Koppen IJN, Velasco-Benítez CA, Benninga MA, Di Lorenzo C, Saps M. Using the Bristol Stool Scale and Parental Report of Stool Consistency as Part of the Rome III Criteria for Functional Constipation in Infants and Toddlers. J Pediatr. 2016; 177: 44-48

17. Velasco-Benítez CA, Sánchez MP, Aragón LE. Realibility of the FINDERS questionnaire in Spanish based on Rome III Criteria for functional gastrointestinal disorders in infants and toddler from Colombia, South America. JPGN. 2015; 61 (Suppl 2): S57

18. Hyman PE, Milla PJ, Benninga MA, Davidson GP, Fleisher DF, Taminiau J. Childhood Functional Gastrointestinal Disorders: Neonate/Toddler. Gastroenterology. 2006; 130: 1519-1526

19. van Tilburg MAL, Rouster A, Silver D, Pellegrini G, Gao J,
Hyman PE. Development and Validation of a Rome III Functional Gastrointestinal Disorders Questionnaire for Infants and Toddlers. JPGN. 2015; 62: 384-386

20. Rouster AS, Karpinski AC, Silver D, Monagas J, Hyman PE. Functional Gastrointestinal Disorders Dominate Pediatric Gastroenterology Outpatient Practice. JPGN. 2016; 62: 847-851

21. Ferreira-Maia AP, Matijasevich A, Wang YP. Epidemiology of functional gastrointestinal disorders in infants and toddlers: A systematic review. World J Gastroenterol. 2016; 22: 6547-6558

22. Di Lorenzo C. Other Functional Gastrointestinal Disorders in Infants and Young Children. JPGN. 2013; 57 (Suppl 1): S36-S38

23. Steutel NF, Benninga MA, Langendam MW, de Kruijff I, Tabbers MM. Reporting Outcome Measures in Trials of Infant Colic. JPGN. 2014; 59: 341-346

24. Szajewska H, Dryl R. Probiotics for the Management of Infantile Colic. JPGN. 2016; 63 (Suppl 1): S22-S24

25. Chogle A, Velasco-Benitez CA, Chanis R, Mejia M, Saps M. Multicountry cross-sectional study found that functional gastrointestinal disorders such as colic and functional dyschezia were common in South American infants. Acta Pædiatr. 2018; 107: 708-713

26. Romanello S, Spiri D, Marcuzzi E, Zanin A, Boizeau P, Riviere S, et al. Association Between Childhood Migraine and History of Infantile Colic. JAMA. 2013; 309: 1607-1612

27. von Baeyer CL, Champion GD. Commentary: multiple pains as functional pain syndromes. J Pediatr Psychol. 2011; 36: 433-437

28. Indrio F, Di Mauro A, Riezzo G, Cavallo L, Francavilla R. Infantile colic, regurgitation, and constipation: an early traumatic insult in the development of functional gastrointestinal disorders in children? Eur J Pediatr. 2015; 174: 841-842

29. Bekkali N, Hamers SL, Reitsma JB, van Toledo L, Benninga M. Infant Stool Form Scale: Development and Results. J Pediatr. 2009; 154: 521-526 